\title{
Overdose Prevention and Naloxone Prescription for Opioid Users in San Francisco
}

\author{
Lauren Enteen, Joanna Bauer, Rachel McLean, Eliza Wheeler,
} Emalie Huriaux, Alex H. Kral, and Joshua D. Bamberger

\begin{abstract}
Opiate overdose is a significant cause of mortality among injection drug users (IDUs) in the United States (US). Opiate overdose can be reversed by administering naloxone, an opiate antagonist. Among IDUs, prevalence of witnessing overdose events is high, and the provision of take-home naloxone to IDUs can be an important intervention to reduce the number of overdose fatalities. The Drug Overdose Prevention and Education (DOPE) Project was the first naloxone prescription program (NPP) established in partnership with a county health department (San Francisco Department of Public Health), and is one of the longest running NPPs in the USA. From September 2003 to December 2009, 1,942 individuals were trained and prescribed naloxone through the DOPE Project, of whom $24 \%$ returned to receive a naloxone refill, and $11 \%$ reported using naloxone during an overdose event. Of 399 overdose events where naloxone was used, participants reported that $89 \%$ were reversed. In addition, $83 \%$ of participants who reported overdose reversal attributed the reversal to their administration of naloxone, and fewer than $1 \%$ reported serious adverse effects. Findings from the DOPE Project add to a growing body of research that suggests that IDUs at high risk of witnessing overdose events are willing to be trained on overdose response strategies and use take-home naloxone during overdose events to prevent deaths.
\end{abstract}

KEYWORDS Overdose, Heroin, Naloxone, Injection drug user

\section{INTRODUCTION}

Drug-related deaths are the leading cause of injury mortality among all US adults aged 35 to $55 .^{1}$ Opioids are one of the most commonly involved substances in single and polydrug use deaths. ${ }^{2}$ Opiate overdose is the single greatest cause of mortality among injection drug users (IDUs) in the USA $^{3}$ and accounts for more than half of all deaths among opiate injectors, far exceeding the proportion due to HIV/AIDS and viral hepatitis. ${ }^{3,4}$ Opiate overdose deaths increased by $529 \%$ between 1990 and 2003 across the USA. ${ }^{2}$ In addition, opioid analgesic-related deaths are among the fastest growing causes of drug poisoning deaths in the USA. ${ }^{1}$

While loss of consciousness following overdose can at times be instantaneous, death is usually the result of cardiac arrest that follows hypoxia, which is the result of

Enteen is with the Masters Entry Program in Nursing, University of California, San Francisco, CA, USA; Bauer and Bamberger are with the Housing and Urban Health, San Francisco Department of Public Health, San Francisco, CA, USA; McLean is with the California Department of Public Health, San Francisco, CA, USA; Wheeler is with the Drug Overdose Prevention and Education Project, Oakland, CA, USA; Huriaux is with the AIDS Office, San Francisco Department of Public Health, San Francisco, CA, USA; Kral is with the RTI International, San Francisco, CA, USA.

Correspondence: Joshua D. Bamberger, Housing and Urban Health, San Francisco Department of Public Health, San Francisco, CA, USA. (E-mail: josh.bamberger@sfdph.org) 
the opiate suppressing the central respiratory drive. ${ }^{5,6}$ The time from initial injection to death typically leaves a $1-3-\mathrm{h}$ window for a witness to intervene. ${ }^{5,6}$ While this window would leave sufficient time for emergency personnel to respond, studies report that emergency medical services (EMS) are activated in fewer than half of overdose events. $^{7-9}$ Although cardiopulmonary resuscitation can be an effective intervention during an overdose event, ${ }^{10}$ opiate overdose can almost universally be reversed by the administration of naloxone-a legal, nonscheduled opioid antagonist that can be quickly administered by intramuscular injection. ${ }^{10-12}$ Naloxone produces no symptoms of dependence or tolerance and, in the absence of narcotics, has no pharmacological activity. ${ }^{13}$ Naloxone is routinely administered by emergency services personnel to revive opiate overdose victims, and serious side effects are rare. ${ }^{14-17}$

Yet, barriers to intervention and overdose reversal remain. Between 1997 and 2000, EMS response was noted in medical examiner's notes for only $26 \%$ of fatal opiate overdoses in San Francisco. ${ }^{8}$ In surveys, IDUs consistently report a high prevalence of witnessing overdose events, ${ }^{9,18-22}$ and in one Bay Area survey, $89 \%$ of participants reported witnessing an overdose event. ${ }^{9}$ However, IDUs also report reluctance to contact EMS as a witness. ${ }^{9,21-25}$ Qualitative research with IDUs indicates that fear of police is a significant barrier to calling emergency services ("9-1-1" in the USA) during an overdose event. ${ }^{21,23}$ IDUs report making other attempts to revive overdose victims without EMS assistance, $, 21,22$ and demonstrate willingness to administer naloxone during an overdose if it was made available to them directly. ${ }^{9,18,23,24}$ This indicates that targeted take-home naloxone prescription and overdose training programs may be an effective intervention to reduce opiate overdose deaths.

In response to increased fatal opiate overdose, community-based programs began distributing naloxone directly to IDUs in Europe in 1995, ${ }^{26}$ and underground programs have been distributing naloxone in the USA since $1999 .{ }^{27}$ Take-home naloxone prescription programs (NPPs) are currently in place in locales throughout the USA, including large-scale NPPs in Chicago, Baltimore, New York City, New Mexico, and Massachusetts. ${ }^{27-29}$ NPPs typically provide overdose response education and naloxone administration training to IDUs and others at high risk of witnessing an opioid overdose, so that participants are able to administer naloxone safely and avert fatalities during overdose events.

Preliminary evaluations of NPPs in several cities have found that overdose response education and naloxone administration training positively affects IDUs' ability to recognize overdose symptoms and identify cases where naloxone is indicated. ${ }^{24,28-30}$ Prospective pilot studies in Los Angeles ${ }^{32}$ and New York ${ }^{23,33}$ and San Francisco ${ }^{30}$ tracked small samples of IDUs who were trained and provided with naloxone. In New York and San Francisco, over half reported using naloxone during 3- or 6-month follow-up periods, and the proportion of participantconfirmed reversals ranged from $74 \%{ }^{32}$ to $100 \% .^{23,30,33}$

Fewer studies have examined outcomes of an NPP over an extended period of time. One longstanding NPP in Chicago reported training 3,500 participants from 2001 to 2005, of whom 319 reported overdose reversals $(9 \%) .{ }^{27}$ A program dispending intranasal naloxone in Massachusetts recently reported that $19 \%$ (74) of 385 trained participants used naloxone after training. ${ }^{34}$

In 2003, the San Francisco Department of Public Health (SFDPH) partnered with a community-based program, the Drug Overdose Prevention and Education Project (DOPE Project) to establish the first health-department sanctioned NPP in the US. Modeled on underground community based NPPs, the DOPE Project was the first NPP to receive staff and support from a county department of public health. 
The goal of the DOPE Project is to integrate overdose prevention education and naloxone distribution into all settings serving people at risk for opioid overdose. ${ }^{31}$

We present evaluation findings for the DOPE Project NPP in San Francisco, from the start of SFDPH partnership in September 2003 through December 2009. Our goal was to examine the number and demographics of trained participants prescribed take-home naloxone, as well as the prevalence of and reasons for receiving naloxone refills among trained participants. We present prevalence of naloxone administration among individuals receiving refills from the DOPE Project and outcomes of naloxone administration, including any negative effects reported and overall proportion of successful reversals.

\section{METHODS}

\section{The San Francisco DOPE Project Intervention}

Since September 2003, DOPE Project staff and SFDPH medical providers have trained and distributed naloxone at sites throughout San Francisco that include syringe exchange programs (SEP), re-entry programs, pain management clinics, methadone maintenance and buprenorphine treatment programs, and single room occupancy (SRO) hotels. The DOPE Project currently conducts trainings and naloxone dispensations approximately eight times per month throughout San Francisco.

Participants are usually recruited and trained while waiting to receive services at clinics, dropping off syringes at SEPs, or in group trainings in SROs and treatment programs. Trainings typically last between 10 and 30 minutes and focus on overdose symptom identification, revival strategies, calling EMS, and administering naloxone (Figure 1). After DOPE Project staff train participants, SFDPH medical providers initiate a medical record (clinical registration) and assign each participant a unique identifier. Providers prescribe and dispense naloxone in two $0.4-\mathrm{mg} / \mathrm{mL}$ vials and two $3-\mathrm{cm}^{3} / \mathrm{mL} 22$-gauge 1 -in muscling syringes along with a rescue breathing mask. All trained participants with unique identifiers and clinical registrations may receive refills of two pre-filled syringes at any subsequent dispensation-when participants use naloxone, lose, or have naloxone confiscated. SFDPH providers do not limit the number of refills trained participants may receive.

\section{Data Collection}

All participants who receive take-home naloxone complete a brief questionnaire immediately following initial training. The questionnaire is voluntary, self-reported, and administered by DOPE Project or SFDPH staff. Information provided includes date of birth, gender, race/ethnicity, primary language, homeless status, and/or current housing.

All participants who receive subsequent refills also complete an additional brief questionnaire. If receiving naloxone following a loss, participants describe "circumstances of loss (e.g., stolen bag, taken by police, etc.).”

Participants who receive refills following naloxone administration complete a brief interview with DOPE Project staff. The standard questionnaire captures information about to whom naloxone was administered (e.g., "girlfriend," "spouse," "friend," "stranger," "self"), and whether participants used other prevention strategies covered in DOPE Project training: sternum rub; awaken victims; call emergency services; rescue breathing; waited with them. Participants are 


\author{
Mechanism of opiate overdose \\ Risk factors for opiate overdose \\ Prevention strategies \\ Recognition \\ Response \\ Calling 9-1-1 \\ Administration of naloxone \\ Rescue breathing \\ Aftercare \\ Naloxone care \\ Logistics and refills
}

FIGURE 1. Components of $10-30-\mathrm{min}$ trainings conducted by DOPE Project staff for all participants receiving take-home naloxone.

also asked whether an ambulance arrived, and to choose from among seven possible outcomes for the event: they [victim] woke up without any help; they woke up because of my help; paramedics came and revived the person [victim]; paramedics came and I do not know what happened next; they [victim] died; do not know; other (specify). Participants report any "negative consequences" of the overdose and naloxone administration that include: arrest of victim or witness; vomiting; harassment by police; harassment by paramedics; seizure; other (specify).

\title{
Analysis
}

All records used in this study were obtained as part of the DOPE Project routine program monitoring and evaluation. We considered all individuals who were trained, prescribed, and assigned a unique identifier (at clinical registration) as participants in the DOPE Project and used a clinical registration database (Microsoft Excel, Seattle, WA, USA) to calculate total number of trained participants, and participant demographics. Participants who reported being homeless, living at a shelter, transitional housing, or street or "couch surfing" were coded as unstably housed.

A separate database of all refills is also maintained by the DOPE Project (Microsoft, Excel, Seattle, WA). Databases were linked by participants' unique identifiers to determine the number of unduplicated participants who received refills following self-reported loss or use of naloxone, as well as the total number of losses and naloxone use reported, the proportion of individuals receiving multiple refills, and the proportion of individuals reporting multiple naloxone use. We excluded from analysis any records of refills where no unique identifier could be linked to an existing clinical registration $(n=37)$.

We coded reports stating that naloxone was taken by police, San Francisco Department of Public Works (DPW) or sheriff's office as confiscation; all reports that naloxone was stolen, lost, or destroyed for any reason was coded as being lost. We use information captured in refill questionnaire to determine outcomes for trained participants who reported administering naloxone. Questionnaires were used to determine proportion of participants who used strategies other than naloxone, including contacting EMS. All events where participants reported that the victim was "revived" after naloxone administration are included here as 
successful reversals-whether or not participants specifically attributed the reversal to naloxone administration. Questionnaires were also used here to report proportion of deaths, unknown outcomes, or any negative effects.

\section{RESULTS}

From September 2003 to December 2009, the DOPE Project and SFDPH medical providers trained and prescribed naloxone to 1,942 unduplicated individuals in San Francisco. The number of new participants increased steadily from 2003 to 2009, averaging 328 per year.

The majority of participants were male $(64 \%)$ and the median age at training was 40 years old (Table 1). Race/ethnicity was only captured for $75 \%$ of participants overall. Of these, $61 \%$ were Caucasian and $18 \%$ were African American. Housing status was reported by $88 \%$ of participants, of whom over half (59\%) reported being homeless or unstably housed (not shown).

Of the 1,942 participants who receive naloxone prescriptions, $24 \%$ returned to receive at least one naloxone refill (Table 2), of whom half returned on more than one occasion to receive multiple refills. Participants requested refills for a variety of reasons, including having naloxone stolen on the street, confiscated in a shelter, or destroyed during unstable housing transition. Of 1,020 refills dispensed, $399(40 \%)$ were provided after participants reported using naloxone during an overdose event,

TABLE 1 DOPE Project participants trained and prescribed 2003-2009 $(n=1,942)$

\begin{tabular}{lll}
\hline & $n$ & $(\%)$ \\
\hline Gender & & \\
Male & 1,239 & $(64)$ \\
Female & 644 & $(33)$ \\
Transgender & 15 & $(1)$ \\
Unknown (not captured) & 44 & $(2)$ \\
Race/ethnicity & & \\
Caucasian/White & 901 & $(46)$ \\
African American/Black & 263 & $(14)$ \\
Latino/a & 131 & $(7)$ \\
Asian/Pacific Islander & 32 & $(2)$ \\
Native American & 35 & $(2)$ \\
More than one race/ethnicity & 53 & $(3)$ \\
Other & 51 & $(3)$ \\
Unknown (not captured) & 476 & $(24)$ \\
Housing status & & $(32)$ \\
Stable housing & 618 & $(46)$ \\
Homeless/unstably housed & a \\
Living in shelter & 893 & $(7)$ \\
Living in transitional housing & 127 & $(15)$ \\
Living on street & 292 & $(12)$ \\
Doubling up or "couch surfing" & 241 & $(3)$ \\
Homeless, no additional housing information & 51 & $(9)$ \\
Unknown housing status & 182 & $(22)$ \\
\hline
\end{tabular}

ancludes both those who answered "yes" to homeless and those reporting living in shelter, transitional housing, or doubling up 
TABLE 2 Participants who received refills from the DOPE Project, 2004-2009

\begin{tabular}{|c|c|c|}
\hline & $n$ & (\%) \\
\hline $\begin{array}{l}\text { Participants who received at least } \\
\text { one naloxone refill, any reason }\end{array}$ & 470 & (24) \\
\hline $\begin{array}{l}\text { Participants who received multiple } \\
\text { refills, any reason }\end{array}$ & 219 & (11) \\
\hline $\begin{array}{l}\text { Participant who received at least one } \\
\text { refill reporting naloxone loss }\end{array}$ & 311 & (16) \\
\hline $\begin{array}{l}\text { Participants who received at least one } \\
\text { refill reporting confiscation }\end{array}$ & 79 & (4) \\
\hline $\begin{array}{l}\text { Participants who received at least one } \\
\text { refill reporting naloxone administration } \\
\text { during an overdose event }\end{array}$ & 215 & (11) \\
\hline $\begin{array}{l}\text { Participants who received multiple } \\
\text { refills reporting naloxone administration } \\
\text { during multiple overdose events }\end{array}$ & 95 & (5) \\
\hline
\end{tabular}

Of 1,942 participants trained 2003-2009

while $49 \%$ were provided after participants reported a loss of naloxone, and $12 \%$ were provided after participants reported confiscation by police, DPW, or sheriff's department (upon admission to jail). Of participants who lost naloxone, $27 \%$ reported that they also used naloxone in response to a subsequent overdose event.

Overall, $11 \%$ of all participants reported using naloxone during an overdose event, and 5\% reported using multiple prescriptions (refills) during more than one overdose event (Table 2). In addition, a small proportion of participants (not shown) reported using multiple naloxone doses to reverse as many as eight separate overdose events. The cumulative number of naloxone-administration events reported to the DOPE Project has risen steadily since 2004 (Figure 2), with an average of 80 events reported annually.

In $83 \%$ of overdose responses reported, participants stated that naloxone administration reversed the overdose. Participants reported successful outcomes (reversal with or without EMS involvement) for $89 \%$ of all overdose events where

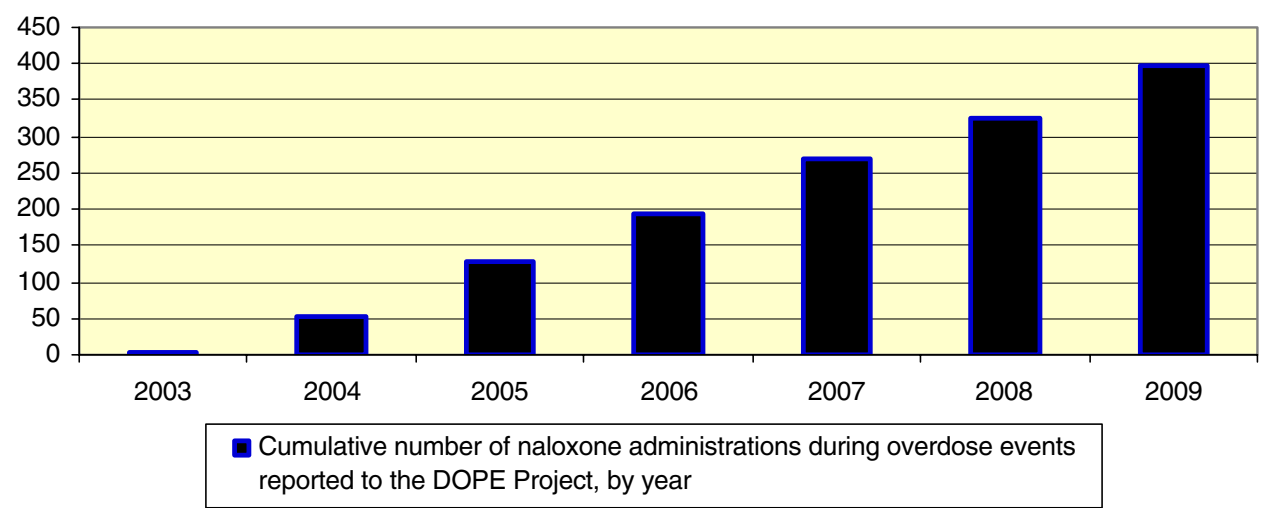

FIGURE 2. Cumulative number of opioid overdose responses with naloxone reported by DOPE Project participants receiving refills, by year, 2003-2009. 
naloxone was used (Table 3), and an additional 3\% of reports indicated that EMS was contacted, but outcome was unknown.

Three-quarters of participants who used naloxone also reported using complementary overdose prevention strategies taught by the DOPE Project such as rescue breathing $(50 \%$, Table 3$)$. However, a minority $(29 \%)$ reported calling emergency services, and only $21 \%$ of reports included indication that EMS arrived. Although only one participant reported arrest following naloxone administration, $13 \%$ of the 84 participants who reported ambulance response also reported some harassment by police or EMS (Table 3).

Serious adverse effects were reported rarely and included several instances of seizures (Table 3). In cases for which participants reported a known outcome, vomiting was the most commonly reported negative effect $(13 \%)$, followed by “anger" or discomfort expressed by victim upon waking (9\%). Three participants $(<1 \%)$ reported witnessing a victim experience symptoms of a seizure following naloxone administration. Victim death was reported by participants in four $(1 \%)$ events where naloxone was used (not shown). In three of these cases, participants reported that the victim had been unconscious for an undetermined amount of time

TABLE 3 Participant reported responses and outcomes of opioid overdose events where naloxone was administered, among participants receiving a naloxone refill from the DOPE Project, 2004-2009 $(n=399)$

\begin{tabular}{|c|c|c|}
\hline & $\mathrm{N}$ & (\%) \\
\hline \multicolumn{3}{|l|}{ Relationship between participant and overdose victim } \\
\hline Participant used naloxone on companion (friend, spouse) & 142 & (36) \\
\hline Participant used naloxone on stranger & 60 & (15) \\
\hline Relationship between participant and victim not reported & 111 & (28) \\
\hline Participant reported naloxone was used on self during overdose & 85 & (21) \\
\hline \multicolumn{3}{|l|}{ Other overdose prevention strategies used in addition to naloxone } \\
\hline Participant reported using any additional strategy & 298 & (75) \\
\hline Sternum rub & 123 & (31) \\
\hline Awaken victim & 127 & (32) \\
\hline Rescue breathing & 199 & $(50)$ \\
\hline Participant reported calling 911 during the overdose event & 116 & (29) \\
\hline Participant reported ambulance response & 84 & (21) \\
\hline \multicolumn{3}{|l|}{ Participant reported outcome of overdose event } \\
\hline Reversed, all reasons & 357 & $(89)$ \\
\hline Reversed due to participant administering naloxone & 333 & (83) \\
\hline Reversed following EMS response & 19 & (5) \\
\hline Naloxone administered, but victim revived by another method & 5 & (1) \\
\hline Victim died & 6 & (2) \\
\hline Outcome Unknown & 36 & (9) \\
\hline \multicolumn{3}{|l|}{ Other adverse outcomes reported } \\
\hline Seizure & 3 & (1) \\
\hline Vomiting & 50 & (13) \\
\hline Victim was angry or "dope sick" & 36 & (9) \\
\hline Arrest & 1 & $(.2)$ \\
\hline EMS/police harassment & 11 & (3) \\
\hline
\end{tabular}

Of 399 overdose events where participants administered naloxone, as reported to DOPE Project by participants receiving naloxone refills, 2004-2009 
before they were found, including one victim who was found unconscious in a large public park.

\section{DISCUSSION}

These findings are the first to examine overall participant demographics for a longstanding NPP, and indicate that the DOPE Project is well targeted to reach participants who are at high risk of overdose. For example, we found that $46 \%$ of those trained by the program identified as unstably housed or homeless, which has been associated with significantly elevated risk of overdose death in previous studies of overdose in San Francisco. ${ }^{29}$ Moreover, by targeting the program in places where there is a high prevalence of IDUs who witness overdose events, the DOPE Project has trained a steady population of newly prescribed participants, many of whom return to receive refills on multiple occasions, which further indicates that they are not only willing to be trained, but also motivated to keep a naloxone supply available for use during an overdose.

We found a substantial proportion of trained participants who reported using naloxone, and the proportion found here was consistent with reports from the Chicago Recovery Alliance, one of the largest NPPs in the USA. ${ }^{27}$ Additional previous studies have focused on prospectively tracked small samples and targeted follow up, ${ }^{23,30,32,33}$ making utilization and reversal rates difficult to compare to our findings. However, that these studies consistently find high proportions of participants reporting naloxone use at follow up may suggest that the proportion of participants who report naloxone utilization at refill is an underestimate of the overall proportion of participants who use naloxone after training.

We found that the majority of individuals who used naloxone during an overdose event also indicated that they used other revival methods taught during DOPE trainings. However, a minority reported contacting EMS. An earlier survey of IDUs in San Francisco found that $62 \%$ reported they would be "less inclined" to contact EMS if they had naloxone. ${ }^{8}$ That $84 \%$ of participants who used naloxone reported successful reversal without EMS intervention may suggest that IDUs did not call EMS because they did not deem it necessary, given successful reversal. Among participants who did not know the outcome of the overdose event, calling EMS was more common. Nevertheless, calling emergency services is an important component of training, and efforts should continue to be made to assess and address IDU barriers to contacting EMS during an overdose event, as well as continuing to provide naloxone education and prescription.

Among participants who reported using naloxone, the majority reported positive and successful experiences. Findings here on rates of success per event reported were within the range found in previous studies. ${ }^{32,34}$ Reports of adverse events were rare $(<1 \%$ reported victim seizure following naloxone administration). In addition, although six deaths were reported, there was no indication that this was due to naloxone administration and may have been cases where individuals had been experiencing the overdose for several hours before a witness arrived. Our findings indicate that IDUs can and will successfully administer naloxone with brief training, intervening during overdose events that may otherwise be fatal.

\section{Limitations}

There were several methodological limitations that we encountered while interpreting our findings. All information is self-reported by participants and could be 
affected by social desirability or recall bias among participants completing the refill questionnaire. Due to participant non-disclosure, missing information about race/ ethnicity and housing status made it difficult to assess any trends in refill receipt or naloxone use by these characteristics. Findings reported about naloxone administration and outcome excluded seven reports where unique identifiers could not be matched to clinical registrations. However, no serious adverse outcomes were reported in excluded records.

Our findings on naloxone use here is limited to participants who received refills. DOPE Project staff made no attempts to actively follow up with other participants. Therefore, we cannot determine an exact proportion of reversal, loss, or confiscation among participants overall. It is similarly difficult to draw conclusions about the actual incidence of adverse effects following naloxone administration. Unfortunately, we did not collect detailed information in our structured questionnaire about the extent of the non-fatal adverse events. As such, we cannot know the extent of the symptoms of seizures, which could have ranged from tonic-clonic activity to slight alterations of consciousness. Individuals with positive experiences may have been more likely to request refills than those with negative experiences using naloxone. However, few adverse effects were reported, and the proportion of reported deaths from unsuccessful reversals was similar to other NPP evaluations. ${ }^{27,32,34}$

\section{CONCLUSION}

This is the first longitudinal evaluation of participants, refill request, naloxone use, and outcomes among IDUs participating in a take-home naloxone prescription program in San Francisco. Participation has grown steadily among individuals at high risk of witnessing overdose events, and findings indicate that participants are motivated to receive refills following naloxone loss or use. Among trained participants who report using naloxone, nine in 10 report positive outcomes. Few serious side effects or deaths were reported. The findings presented here add to a growing body of evidence that supports the positive impact of NPPs as an intervention to prevent potentially fatal overdose events.

\section{ACKNOWLEDGEMENTS}

The authors are grateful to the staff and trainers of the DOPE Project and SFDPH medical providers for their contribution to management of the program. Assistance with data collection and management was provided by DOPE Project staff and SFDPH medical providers. This manuscript is dedicated to the memory of Peter Morse, Ph.D., whose extraordinary work improved the lives of so many of us.

OPEN ACCESS This article is distributed under the terms of the Creative Commons Attribution Noncommercial License which permits any noncommercial use, distribution, and reproduction in any medium, provided the original author(s) and source are credited.

\section{REFERENCES}

1. Warner M, Chen LH, Makuc DM. Increase in fatal poisonings involving opioid analgesics in the United States, 1999-2006. NCHS data brief, no. 22. Hyattsville, MD: National Center for Health Statistics; 2009. 
2. Substance Abuse and Mental Health Services Administration OAS. Drug Abuse Warning Network, 2003: Area Profiles of Drug-Related Mortality. Rockville, MD: U.S. Department of Health and Human Services; 2005.

3. Latkin CA, Hua W, Tobin K. Social network correlates of self-reported non-fatal overdose. Drug Alcohol Depend. 2004; 73(1): 61-67.

4. Sherman SG, Cheng Y, Kral AH. Prevalence and correlates of opiate overdose among young injection drug users in a large U.S. city. Drug Alcohol Depend. 2007; 88(2-3): 182187.

5. Sporer KA. Acute heroin overdose. Ann Intern Med. 1999; 130(7): 584-590.

6. Warner-Smith M, Darke S, Lynskey M, Hall W. Heroin overdose: causes and consequences. Addiction. 2001; 96(8): 1113-1125.

7. Darke S, Zador D. Fatal heroin 'overdose': a review. Addiction. 1996; 91(12): 1765-1772.

8. Davidson PJ, McLean RL, Kral AH, Gleghorn AA, Edlin BR, Moss AR. Fatal heroinrelated overdose in San Francisco, 1997-2000: a case for targeted intervention. J Urban Health. 2003; 80(2): 261-273.

9. Seal KH, Downing M, Kral AH, et al. Attitudes about prescribing take-home naloxone to injection drug users for the management of heroin overdose: a survey of street-recruited injectors in the San Francisco Bay Area. J Urban Health. 2003; 80(2): 191-301.

10. Baca CT, Grant KJ. Take-home naloxone to reduce heroin death. Addiction. 2005; 100 (12): 1823-1831.

11. Dixon P. Managing acute heroin overdose. Emerg Nurse. 2007; 15(2): 30-35.

12. Sporer KA. Strategies for preventing heroin overdose. BMJ. 2003; 326(7386): 442-444.

13. Chamberlain JM, Klein BL. A comprehensive review of naloxone for the emergency physician. Am J Emerg Med. 1994; 12(6): 650-660.

14. Coffin PO, Fuller C, Vadnai L, Blaney S, Galea S, Vlahov D. Preliminary evidence of health care provider support for naloxone prescription as overdose fatality prevention strategy in New York City. J Urban Health. 2003; 80(2): 288-290.

15. Belz D, Lieb J, Rea T, Eisenberg MS. Naloxone use in a tiered-response emergency medical services system. Prehosp Emerg Care. 2006; 10(4): 468-471.

16. Buajordet I, Naess AC, Jacobsen D, Brors O. Adverse events after naloxone treatment of episodes of suspected acute opioid overdose. Eur J Emerg Med. 2004; 11(1): 19-23.

17. Sporer KA, Kral AH. Prescription naloxone: a novel approach to heroin overdose prevention. Ann Emerg Med. 2007; 49(2): 172-177.

18. Lagu T, Anderson BJ, Stein M. Overdose among friends: drug users are willing to administer naloxone to others. J Subst Abuse Treat. 2006; 30(2): 129-133.

19. Davidson PJ, Ochoa KC, Hahn JA, Evans JL, Moss AR. Witnessing heroin-related overdoses: the experiences of young injectors in San Francisco. Addiction. 2002; 97(12): 1511-1516.

20. Ochoa KC, Hahn JA, Seal KH, Moss AR. Overdosing among young injection drug users in San Francisco. Addict Behav. 2001; 26(3): 453-460.

21. Dettmer K, Saunders B, Strang J. Take home naloxone and the prevention of deaths from opiate overdose: two pilot schemes. BMJ. 2001; 322(7291): 895-896.

22. Tracy M, Piper TM, Ompad D, et al. Circumstances of witnessed drug overdose in New York City: implications for intervention. Drug Alcohol Depend. 2005; 79(2): 181-190.

23. Galea S, Worthington N, Piper TM, Nandi VV, Curtis M, Rosenthal DM. Provision of naloxone to injection drug users as an overdose prevention strategy: early evidence from a pilot study in New York City. Addict Behav. 2006; 31(5): 907-912.

24. Strang J, Manning V, Mayet S, et al. Overdose training and take home naloxone for opiate users: prospective cohort study of impact on knowledge and attitudes and subsequent management of overdoses. Addiction. 2008; 103(10): 1648-1657.

25. Tobin K, Davey MA, Latkin CA. Calling emergency medical services during drug overdose: an examination of individual, social and setting correlates. Addiction. 2005; 100(3): 397-404. 
26. Ronconi S. Prevention of overdoses among current heroin users in Torino, Italy, for the period 1995-1998. Paper presented at: Preventing Heroin Overdose: Pragmatic Approaches; January 13-14, 2000; Seattle, WA.

27. Maxwell S, Bigg D, Stanczykiewicz K, Carlberg-Racich S. Prescribing naloxone to actively injecting heroin users: a program to reduce heroin overdose deaths. J Addict Dis. 2006; 25(3): 89-96.

28. Green TC, Heimer R, Grau LE. Distinguishing signs of opioid overdose and indication for naloxone: an evaluation of six overdose training and naloxone distribution programs in the United States. Addiction. 2008; 103(6): 979-989.

29. Worthington N, Markham Piper T, Galea S, Rosenthal D. Opiate users' knowledge about overdose prevention and naloxone in New York City: a focus group study. Harm Reduct J. 2006; 3: 19.

30. Seal KH, Thawley R, Gee L, et al. Naloxone distribution and cardiopulmonary Resuscitation Training for injection drug users to prevent heroin overdose death: a pilot intervention study. J Urban Health. 2005; 82: 303-311.

31. Seal KH, Kral AH, Gee L, et al. Predictors and prevention of nonfatal overdose among street-recruited injection heroin users in the San Francisco Bay Area, 1998-1999. Am J Public Health. 2001; 91(11): 1842-1846.

32. Wagner K, Valente T, Casanova M, et al. Evaluation of an overdose prevention and response training programme for injection drug users in the Skid Row area of Los Angeles, CA. Int J Drug Policy. 2009. doi:10.1016/j.drugpo.2009.01.003.

33. Piper TM, Stancliff S, Rudenstine S, et al. Evaluation of a naloxone distribution and administration program in New York City. Subst Use Misuse. 2008; 43(7): 858-870.

34. Doe-Simkins M, Walley AY, Epstein A, Moyer P. Saved by the nose: bystanderadministered intranasal naloxone hydrochloride for opioid overdose. Am J Public Health. 2009; 99(5): 788-791. 